\title{
Von F(acebook) bis X(ing)
}

Der Begriff des sozialen Netzwerkes stammt aus der Ethnosoziologie, im modernen Web 2.0 beschreibt er hingegen Plattformen, die ihren Anwendern ein Netzwerk aus Bekanntschaften als virtuelles Interaktionsgeflecht anbieten. Soziale Netzwerke und die in ihnen abgelegten persönlichen Profile haben heute einen wichtigen Stellenwert bekommen: Sie repräsentieren die virtuellen Identitäten der Nutzer im Internet.

Für soziale Netzwerke gibt es, wie auch die in den Beiträgen in diesem Heft verwendeten Begriffe zeigen, unterschiedliche Bezeichnungen, und auch hinsichtlich ihrer Nutzung zeigt sich eine enorme Vielfalt: Von der Foto-Plattform Flickr über das Schüler-Netzwerk schueler$V Z$ bis hin zu Netzwerken für die berufliche Weiterentwicklung im Rahmen von Xing sind verschiedenste Plattformen nutzbar.

Vielfalt bringt aber auch Risiken mit sich und dies nicht nur, weil mit mehreren Mitgliedschaften auch die Menge der veröffentlichten Daten steigt. Eine wichtige Fragestellung in diesem Kontext ist daher: Wie können soziale Netzwerke und ihre positiven Aspekte weiterhin genutzt und die eigene Privatsphäre in ein vertretbares Verhältnis dazu gesetzt werden? Die Beiträge im Schwerpunkt dieses Heftes versuchen Antworten auf diese Fragestellung zu geben.

\section{Das Spektrum der behandelten Aspekte ist breit}

- Der Beitrag Datenschutzkonformes Social Networking: Clique und Scramble! von Leif-Erik Holtz stellt einen Lösungsansatz vor, mit dem die Daten in sozialen Netzwerken durch feingranulare Zugriffsrechte und Verschlüsselung vor unliebsamer Einsicht durch Angreifer, aber auch den Betreiber(!) geschützt werden können.

- Das Thema Datenschutz Compliance in Sozialen Netzwerk Anwendungen greift Stefan Weiss in seinem Beitrag auf. Er diskutiert Voraussetzungen und Anforderungen, die erfüllt werden müssen, um eine technische Umsetzbarkeit der Compliance gewährleisten zu können.

- Kontrolle über eigene Daten in sozialen Netzwerken ist ein wichtiger Aspekt, den Johannes Mainusch und Christian Burtchen diskutieren. Sie werfen dabei auch die Frage auf, wie Nutzer sozialer Netzwerke die Kontrolle über ihre Daten erhalten können.

- Facebook, Web 2.0 und ökonomische Überwachung wird von Christian Fuchs beleuchtet. Das Thema der ökonomischen Überwachung im Internet und in sozialen Netzwerken bietet viel Diskussions- und Konfliktstoff - eine entsprechende Analyse ist daher ein Muss und zeigt zudem interessante Details auf.

- Der Beitrag Persönlichkeit 2.0 vom Autorenteam rund um Boris Egloff widmet sich der Frage, welche Genauigkeit Persönlichkeitsurteile aus Daten sozialer Netzwerke haben. Eine Fra$\mathrm{ge}$, die durch die steigende Informationsgewinnung aus dem Web $2.0 \mathrm{im}$ Rahmen von Bewerbungsgesprächen immer wichtiger wird.

- Die Rolle des Rechts in einer durch Web 2.0 dominierten Welt wird von den Autoren Thomas Hoeren und Gootfried Vossen diskutiert. Sie zeigen dabei auf, dass ohne eine interdisziplinäre Zusammenarbeit keine adäquaten Lösungen im Problembereich des Web 2.0 zu erwarten sind.

- Löschen durch Verschlüsselung beschreibt einen kryptografischen Ansatz zum Löschen von Daten. Ulrich Greveler und Christoph Wegener zeigen in ihrem Beitrag, wie damit auch Unternehmen mit komplexen Informationsstrukturen nicht auf Sperren zurück greifen müssen, um datenschutzgerecht zu löschen.

- Datenverlust und die Pflicht zur Öffentlichkeit ist eine der Folgen der Novelle des Bundesdatenschutzgesetzes. Stefan Ernst diskutiert in seinem Beitrag die Neuerungen durch § 42a BDSG und hinterfragt dabei kritisch deren Sinn und mögliche Auswirkungen in der Praxis.

- Three Years Hacker Paragraph - A review ist ein Resumé der Entwicklungen rund um § 202c des Strafgesetzbuches. Mark Seeger zeigt in seinem Bericht auf, dass die große Aufregung um die Auswirkungen des so genannten Hackerparagrafen nahezu umsonst war.

Zusammen mit dem gesamten Herausgeberteam wünschen wir Ihnen als Gastherausgeber eine spannende Lektüre. Wir haben uns mit den vorliegenden Beiträgen um eine interessante und repräsentative Auswahl bemüht und hoffen, dass auch diese Ausgabe der DuD Ihnen, verehrte Leserinnen und Leser, viele Anregungen gibt. 